\title{
Assessment of Utility of Ductal Lavage and Ductoscopy in Breast Cancer-A Retrospective Analysis of Mastectomy Specimens
}

\author{
Sunil Badve, M.B, B.S., M.D. (Path), F.R.C.Path, Elizabeth Wiley, M.D., Norma Rodriguez, M.D. \\ Northwestern University Medical School, Chicago, Illinois (SB, EW, NR) and Indiana University School of \\ Medicine, Indianapolis, Indiana (SB)
}

Early detection of breast lesions continues to be an important goal in the management of breast cancer. At present, mammographic imaging in addition to physical examination is the main screening method for the detection of cancer. Fiberoptic ductoscopy and duct lavage are being recently used to evaluate patients at risk for breast cancer. Both techniques examine the nipple and central duct area to identify intraductal lesions. In this study, we examined the frequency of involvement of these structures in mastectomy specimens as a surrogate marker to estimate the utility of these methods in breast cancer patients. The presence and type of involvement of the nipple and central duct area was retrospectively evaluated in 801 mastectomy specimens from a 4 -year period that had been performed for infiltrating or in situ carcinoma. Atypical proliferation or cells, when seen in the ducts of this region, was considered as evidence of nipple involvement, even if definite evidence of malignancy was lacking. The review of 801 mastectomies showed nipple and central duct involvement in $179(22 \%)$ cases. Among the 665 cases of infiltrating carcinoma, $17 \%$ did not have an intraductal component. The relative rarity of nipple and central duct in mastectomy specimens and the lack of an in situ component in many cases raise questions about the utility of fiberoptic ductoscopy and duct lavage as methods for screening of breast cancer. Additionally, as these methods examine only 1-2

Copyright (C) 2003 by The United States and Canadian Academy of Pathology, Inc.

VOL. 16, NO. 3, P. 206, 2003 Printed in the U.S.A.

Date of acceptance: January 6, 2003.

Address reprint requests to: Sunil Badve, M.B., B.S., M.D. (Path), F.R.C.Path, Division of Surgical Pathology, Indiana School of Medicine, 635 Barnhill Drive, Indianapolis, IN 46202; fax: 317-274-5346; e-mail: sunilb@pol.net.

DOI: $10.1097 / 01 . M P .0000056985 .01062 . E 7$ ducts of the 15-20 ducts that open at the nipple, they might fail to detect focal abnormalities.

KEY WORDS: Ductal lavage, Fiberoptic ductoscopy, Nipple involvement.

Mod Pathol 2003;16(3):206-209

Breast cancer is the most frequent type of cancer in women and will affect 1 in 12 women during their lifetime (1). Physical examination and mammographic imaging are the currently used methods for screening for breast cancer. Although larger cancers are easily detected by these methods, smaller tumors can be missed, especially if they arise in densely fibrotic breast tissue. The combination of these screening methods with targeted biopsies ultimately has resulted in earlier detection and treatment of breast cancers. This not only improves patient survival but also decreases the morbidity associated with the disease. Unfortunately, we are still unable to detect a significant number of earlystage cancers. It is for this reason that there is an immense interest in developing new methods for breast cancer screening that would permit detection of carcinoma at an earlier stage, as well as precancerous lesions. Duct lavage and the fiberoptic ductoscopy system are two such methods being evaluated for early detection of breast disease.

Duct lavage and fiberoptic ductoscopy involve evaluation of the ductal system of the breast for detection of intraductal carcinomas and precursor lesions such as atypical ductal hyperplasia. At present, breast cancer is believed to originate within the terminal duct lobular units $(2,3)$ through a succession of events involving and ductal carcinoma in situ $(4,5)$. Therefore, visual and/or cytological examination of these structures would permit identification of abnormal lesions before the development of invasive disease.

Ductal lavage is accomplished with nipple aspiration using a 20-mL syringe (6). If fluid is obtained, the fluid-yielding duct is identified and cannulated. 
A microcatheter is advanced approximately $1.5 \mathrm{~cm}$ into the nipple duct orifice, and the duct is lavaged with normal saline and the lavage effluent collected for cytologic examination (7). Ductal lavage has been shown to retrieve a significantly larger number of cells than nipple aspiration alone (7).

Fiberoptic ductoscopy involves the cannulation of a duct in the nipple identified by the presence of a discharge. It consists of a silica fiberscope, a light source, and an image recorder for recording the observed image. Fiberoptic ductoscopy permits the direct visualization of lesions in the major ducts and simultaneously records distances from the nipple orifice to the proximal and distal borders of the lesion. An outer air channel of the fiberscope permits the installation and irrigation of saline washings and retrieval of cells from the ductal system of the breast (8).

Although both methods seem to offer useful information about the ductal system, there are limitations to their use. Both methods examine only $1-2$ ducts and leave the remaining, approximately 1318 , ducts $(9,10)$ that open at the nipple of each breast unexamined. Although fiberoptic ductoscopy permits direct visualization of the lactiferous duct, lactiferous sinus, and the segmental duct and its main branches (up to three divisions), its capability for direct observation of lesions in smallercaliber ducts and ducts at the periphery of the breast is limited by the outer diameter $(0.72 \mathrm{~mm})$ of the scope (8) and the complex branching pattern of the ducts $(9,10)$

In addition, the ductal washing/lavage used by both methods for retrieving intraductal cells for cytological retrieves only a third of the fluid originally infused (7). Therefore, there is a considerable fraction of fluid and cells that remain trapped, probably within the smaller, more distally located ducts. Thus the highest probability of detecting cancer or atypical cells by these methods occurs when the neoplasia involves the nipple or central ducts. In this retrospective study of mastectomies, we examine the nipple central duct disease as a surrogate marker for evaluating the efficacy of fiberoptic ductoscopy and ductal lavage as screening methods for detecting breast cancer.

\section{MATERIALS AND METHODS}

A total of 811 consecutive mastectomies performed at Northwestern Memorial Hospital over a period of 5 years were selected for the analysis. Ten mastectomy specimens performed for Phylloides tumors or as a prophylactic procedure (5 cases each) were excluded from the study. Mastectomies in the remaining cases were performed for infiltrating carcinoma, duct carcinoma in situ, or both.
The type of nipple central duct involvement was noted in each of these cases. Nipple central duct involvement was assessed by microscopic examination of the entire nipple, nipple base, and central duct region distal to the nipple base (minimum, 5 blocks, if no gross disease). Involvement of any of these major ducts by noninvasive disease, that is, duct carcinoma in situ, lobular intraepithelial neoplasia or pagetoid duct carcinoma in situ was considered nipple central duct involvement. Atypical proliferation or cells, when seen in the ducts of this region, was also considered as evidence of nipple involvement (and classified as ductal carcinoma in situ), even if definite evidence of malignancy was lacking. The reason for doing this was that these cells, if shed into the ductal system could give rise to positive findings on ductal lavage.

The presence of invasive disease (i.e., infiltrating ductal carcinoma, infiltrating mixed ductal/lobular carcinoma, or infiltrating lobular carcinoma with or without lymphatic invasion located within these selected blocks) was also considered as evidence of nipple central duct involvement.

\section{RESULTS}

The review process identified 801 patients who had undergone mastectomy for breast carcinoma. The type of disease within the breast was duct carcinoma in situ in 136 patients (17\%) and infiltrating carcinoma in 665 patients. The vast majority of invasive tumors were invasive ductal carcinomas (513/665). In 116 of these 665 (17\%) cases with invasive tumors, an in situ component was not identified.

Involvement of the nipple and central duct region was identified in 179 of the 801 mastectomies (22\%). Nipple central duct was seen in $22 \%$ (115/ 522) of all infiltrating carcinoma cases that had duct carcinoma in situ; 32\% (7/22) in cases of infiltrating carcinoma with lobular intraepithelial neoplasia; in $26 \%(5 / 19)$ of lobular carcinoma cases with both duct carcinoma in situ and lobular intraepithelial neoplasia; and in $22 \%(22 / 102)$ of cases of infiltrating carcinoma without an in situ component. Nipple central duct was noted in only $16 \%$ of cases of infiltrating ductal carcinoma. The frequency of nipple central duct was higher in nonductal carcinomas. Nipple central duct was seen in $43 \%$ of lobular carcinoma and $33 \%$ of infiltrating mixed ductal/ lobular carcinoma cases.

The distribution of cases by type of nipple central duct lesion is presented in Table 1 . Noninvasive nipple central duct was seen in 105 cases. In 62 cases, this involvement was in the form of duct carcinoma in situ, whereas in 33 cases, the ducts were partially involved by pagetoid spread of ma- 
TABLE 1. Distribution of Cases by Type of Nipple Central Duct Lesion

\begin{tabular}{lc}
\hline \multicolumn{1}{c}{ Type of Lesion } & Number of Cases \\
\hline Noninvasive & 105 \\
Total & 62 \\
Duct carcinoma in situ & 10 \\
Lobular intraepithelial neoplasia & 33 \\
Pagetoid duct carcinoma in situ & \\
Invasive & 74 \\
Total & 41 \\
Infiltrating ductal carcinoma & 10 \\
Infiltrating mixed ductal/lobular & \\
carcinoma & 9 \\
Lobular carcinoma & 14 \\
Lymphatic Invasion & \\
\hline
\end{tabular}

lignant cells. Involvement by lobular intraepithelial neoplasia was seen in only 10 cases, a reflection of the paucity of lobules in this locale.

\section{DISCUSSION}

In our study of 801 mastectomy cases, we found that $14 \%$ of the ICs did not have an in situ component. The frequency of this finding was lower than that reported in a study undertaken by the National Surgical Adjuvant Breast and Bowel Project (NSABP) (11), which examined almost 1000 mastectomies and found the absence of an in situ component in $>30 \%$ of the cases. Whether the lack of a demonstrable in situ component is a result of it being overrun by the invasive process or due to its genuine absence is a matter of speculation. Because both ductal lavage and fiberoptic ductoscopy depend on the existence of an intraductal component, these procedures would have not been able to detect $14-30 \%$ of these reported cases of breast cancer.

Most of the carcinomas found in our study did not exhibit nipple central duct. The carcinomas in our mastectomy specimens appeared to be located in more peripheral areas of the breast. As the involvement of the ducts can be focal, we opted to use a very broad definition for nipple central duct in our study. Nevertheless, $78 \%(622 / 801)$ of women with cancers undergoing mastectomies did not have nipple central duct. Therefore, it is unlikely that ductoscopy, which can visualize only the first few divisions of the ducts, would be able to identify lesions located in these distal ducts and lobules.

Whether ductal lavage can retrieve a representative cell population of the entire duct/lobular unit, and in particular, cells from peripherally located lesions, is currently not known. Shen et al. (8) performed ductoscopy on 259 patients with nipple discharge and compared the visual findings with cytological washings and in some cases with subsequent histopathology. The positive and negative predictive values for cytologic analysis alone for the presence or absence of an intraductal lesion were $72 \%$ and $50 \%$, as compared with $83 \%$ and $82 \%$ by ductoscopy. Additionally, Shen et al. (8) found that the cells retrieved by the ductal washing used by this method did not represent lesional tissue, even when the lesions were close to the nipple and could be visualized by ductoscopy. In a similar study, Lawler et al. (12) performed ductoscopy in 278 cases of nipple discharge and compared ductal flush cytologic findings with histology in 138 patients. Only $11.1 \%$ of the 27 malignancies diagnosed histologically were identified as malignant by cytologic examination of the ductal washing. Histological identification of intraductal proliferative changes or risk markers of malignancy, such as atypical duct hyperplasia or lobular intraepithelial neoplasia, were found in $88.4 \%$ of cases where cytologic atypia was seen and in $69.6 \%$ cases with normal cytologic findings. This study showed that ductal lavage seems to be specific but not sensitive for the diagnosis of carcinoma and intraductal proliferative changes.

In summary, in this study we evaluated the presence of disease in the nipple and central ducts region as a surrogate marker to evaluate the efficacy of ductal lavage and ductoscopy in detecting neoplasia. A significant number of patients (15-30\%) undergoing mastectomy for breast cancer do not have an intraductal component. Because ductal lavage and ductoscopy can only retrieve cells from within the ducts, neither of these procedures would be able to detect these lesions. Samples obtained by both techniques are derived from a limited number of larger ducts (1-2 ducts/breast), leaving the majority of the breast unexamined. Additionally, neither method may be able to obtain representative samples from the peripheral ducts where precursor and cancerous lesions originate. These findings suggest that fiberoptic ductoscopy and ductal lavage are not good methods for detecting most forms of breast cancers.

\section{REFERENCES}

1. Greenlee RT, Hill-Harmon MB, Murray T, Thun M. Cancer statistics, 2001. CA Cancer J Clin 2001;51(1):15-36.

2. Wellings SR, Jensen HM, Marcum RG. An atlas of subgross pathology of the human breast with special reference to possible precancerous lesions. J Natl Cancer Inst 1975;55(2): 231-73.

3. Wellings S. A hypothesis of the origin of human breast cancer from terminal ductal lobular unit. Pathol Res Pract 1980; 166:515-35.

4. van Diest P. Ductal carcinoma in situ in breast carcinogenesis. J Pathol 1999;187:383-4.

5. Lakhani SR. The transition from hyperplasia to invasive carcinoma of the breast. J Pathol 1999;187(3):272-8.

6. Dooley WC, Veronesi U, Elledge R, O’Shaughnessy J, Ljung B, Arias R. Detection of premalignant and malignant breast cells by ductal lavage. Obstet Gynecol 2001;97(4 Suppl 1):S2. 
7. Dooley WC, Ljung BM, Veronesi U, Cazzaniga M, Elledge RM, O'Shaughnessy JA, et al. Ductal lavage for detection of cellular atypia in women at high risk for breast cancer. J Natl Cancer Inst 2001;93(21):1624-32.

8. Shen KW, Wu J, Lu JS, Han QX, Shen ZZ, Nguyen M, et al. Fiberoptic ductoscopy for breast cancer patients with nipple discharge. Surg Endosc 2001;15(11):1340-5.

9. Ohtake T, Kimijima I, Fukushima T, Yasuda M, Sekikawa K, Takenoshita S, et al. Computer-assisted complete threedimensional reconstruction of the mammary ductal/lobular systems: implications of ductal anastomoses for breastconserving surgery. Cancer 2001;91(12):2263-72.
10. Gray H, Bannister L, Berry M, Williams P. Mammary glands—splanchnology. In: Bannister L, editor. Gray's anatomy: the anatomical basis of medicine \& surgery. 38th ed. London: University of London; 1995.

11. Fisher ER, Gregorio RM, Fisher B, Redmond C, Vellios F, Sommers SC. The pathology of invasive breast cancer. A syllabus derived from findings of the National Surgical Adjuvant Breast Project (protocol no. 4). Cancer 1975;36(1):185.

12. Lawler M, Newton A, Levin E, Mosher R. Cytologic evaluation of duct washings does not reliably detect lactiferous duct pathology. Breast Cancer Res 2001;69(3):297. 